\title{
Cancer screening in Australia: successes, challenges and future directions
}

\author{
Sanchia Aranda $a^{a, c}$ and David C Currow ${ }^{b}$
}

a Cancer Council Australia, Sydney, NSW

b Cancer Institute NSW, Sydney, Australia

c Corresponding author: sanchia.aranda@ cancer.org.au

\section{Article history}

Publication date: July 2019

Citation: Aranda S, Currow DC. Cancer screening in Australia: successes, challenges and future directions. Public Health Res Pract. 2019;29(2):e2921909. https://doi.org/10.17061/phrp2921909
Early diagnosis of cancer is a significant contributor to long-term survival and reduced cancer mortality. Success of a cancer screening program is dependent on there being a latent or early stage of the disease, an acceptable means of finding the cancer or precancerous condition early, and an acceptable treatment available that can demonstrably change the expected outcome. Finding cancers early can contribute to improved quality of cancer survivorship and reduce overall health system costs. When precancerous lesions can be identified and removed, screening also has the potential to lower the incidence of cancer. This issue of Public Health Research \& Practice explores the performance of Australia's cancer screening programs, and how screening may be improved or new approaches to screening developed in the future.

Australia has three population screening programs - for cervical, breast and bowel cancer - and each program faces challenges. All three programs experience lower-than-desired participation rates, especially in communities that are hard to reach. The potential of the National Bowel Cancer Screening Program to reduce the incidence and mortality from bowel cancer and lower healthcare costs has been demonstrated in the Australian setting, and Ee and St John ${ }^{1}$ strongly argue that we must now increase participation for the program to deliver on its promise. Improving participation is possible, as shown by Lotfi-Jam et al. ${ }^{2}$ in this issue, but this requires ongoing investment in public awareness of the program benefits.

As with any population screening, all three programs also face challenges in balancing benefits, harms and cost-effectiveness. Modelling through microsimulation is an exciting new way of informing any potential changes to our screening programs. This approach has been used to inform government policy on cervical and bowel cancer screening in Australia. The role of modelling is explored both by Lew et al. ${ }^{3}$ for Australia's current programs, and by Getaneh et al. ${ }^{4}$ as it might relate to future screening in prostate cancer, where considerations also need to balance concerns about overtreatment.

Screening is, by definition, a population-level intervention, but the onesize-fits-all approach limits our ability to tailor screening to best suit individuals with varying levels of risk. Emerging knowledge of risk factors opens the door for risk-stratified approaches. However, developing these approaches is not straightforward, and risk-based screening will make both the system and the messaging to the public more complicated. Australia has taken the bold step of renewing its cervical screening program to take better account of individual-level risk factors. However, the changes have challenged our complex systems of care, and the capacity to effectively prepare the workforce and eligible women in ways that retain trust in the program. 
The lessons learnt from this change are explored by Smith et al. ${ }^{5}$ and will have implications for any future changes to other programs.

The possibility of a risk-stratified approach is now being explored for potential application within the Australian breast cancer screening program. As knowledge grows about the role of risk factors such as genomic alterations, it may be time to consider applying risk stratification within the program. A potential approach to achieving this is outlined by Nickson et al. ${ }^{6}$

The potential for earlier diagnosis of other cancers through screening is an important consideration. For skin and prostate cancers, the Australian health system already funds opportunistic clinical evaluation, but with no ability to determine the benefits, harms or costeffectiveness of this approach. The large burden of lung cancer incidence and its ensuing mortality cries out for earlier diagnosis, as does the rising burden of liver cancer in our community. The question of what to do about individuals at higher risk of a range of cancers, such as people with Lynch syndrome, challenges our current systems. These issues are explored by a range of authors in the first perspective paper ${ }^{7}$ in this edition.

The overall theme of this issue is that for the benefits of screening to be maximised, we must be prepared to engage in ongoing analysis of program effectiveness, and be open to considering program changes to balance benefits, harms and costs. To do this well, we must invest in linked datasets that enable more complex understandings of the varying impacts of early diagnosis and improvements in treatment on mortality and survival across our community. Throughout this issue, the challenges of effective screening for hard-to-reach populations, such as Aboriginal and Torres Strait Islander people, are raised but not solved. If we are to address the rising gap in outcomes between groups in our community, we must be able to understand the nature and root causes of these variations.

We hope you enjoy this themed issue on cancer screening. Our thanks to the Guest Editors, Karen Canfell, David Roder and Paul Grogan, as well as all the authors who have contributed papers. Thanks too to the many peer reviewers that allowed us to bring you this unique and forward-thinking set of high-quality papers, offering insights into the challenges and future directions for cancer screening in Australia.

\section{Acknowledgements}

This themed issue of Public Health Research \& Practice was supported by and developed in partnership with Cancer Institute NSW and Cancer Council Australia.

\section{Peer review and provenance}

Internally peer reviewed, commissioned.

\section{Competing interests}

In her role as Chief Executive Officer, Cancer Council Australia, SA is involved in advocating for national screening programs and in promoting public awareness of the programs. In his role as Chief Cancer Officer, NSW, and Chief Executive Officer, Cancer Institute NSW, DC oversees the NSW cervical, breast and bowel screening programs.

\section{Author contributions}

SA and DC contributed equally to writing and editing the article.

\section{References}

1. Ee HC, St John J. The National Bowel Cancer Screening Program: time to achieve its potential to save lives. Public Health Res Pract. 2019;29(2):e2921915.

2. Lotfi-Jam KL, O'Reilly CL, Feng CS, Wakefield MA, Durkin $\mathrm{S}$, Broun $\mathrm{KH}$. Increasing bowel cancer screening participation: integrating population-wide, primary care and more targeted approaches. Public Health Res Pract. 2019;29(2):2921916

3. Lew J-B, Feletto E, Wade S, Caruana M, Kang Y-J, Nickson $\mathrm{C}$, et al. Benefits, harms and cost-effectiveness of cancer screening in Australia: an overview of modelling estimates. Public Health Res Pract. 2019;29(2):e2921913.

4. Getaneh AM, Heijnsdijk EAM, de Koning HJ. The role of modelling in the policy decision making process for cancer screening: example of prostate specific antigen screening. Public Health Res Pract. 2019;29(2):e2921912.

5. Smith M, Hammond I, Saville M. Lessons from the renewal of the National Cervical Screening Program in Australia. Public Health Res Pract. 2019;29(2):e2921914.

6. Nickson C, Velentzis LS, Brennan P, Mann GB, Houssami $\mathrm{N}$. Improving breast cancer screening in Australia: a public health perspective. Public Health Res Pract. 2019;29(2):e2921911.

7. Weber MF, Marshall HM, Rankin N, Duffy S, Fong KM, Dunlop K, et al. Cancer screening in Australia: future directions in melanoma, Lynch syndrome, and liver, lung and prostate cancers. Public Health Res Pract. 2019;29(2):e2921910

\section{Copyright: (cc) $\$$}

(C) 2019 Aranda and Currow. This article is licensed under the Creative Commons Attribution-NonCommercial-ShareAlike 4.0 International Licence, which allows others to redistribute, adapt and share this work non-commercially provided they attribute the work and any adapted version of it is distributed under the same Creative Commons licence terms. See: www.creativecommons.org/licenses/by-nc-sa/4.0/ 